J. Clin. Chem. Clin. Biochem.

Vol. 18, 1980, pp. 271-273

\title{
Zur Aktivitätsbestimmung der Sialyltransferase im menschlichen Serum
}

\author{
Von A. Wilhelm, P.-G. Berge und H. Schriewer
}

Aus dem Institut für Arterioskleroseforschung an der Westfälischen-Wilhelms-Universität Münster und der Medizinischen Klinik und Poliklinik Abteilung B (Direktor: Prof. Dr. U. Gerlach) der Westfälischen Wilhelms-Universität Münster

(Eingegangen am 24. September 1979/31. Januar 1980)

Zusammenfassung: Die Aktivität der Sialyltransferase im Serum wurde durch einen radiochemischen Assay mit desialyliertem Fetuin als Akzeptor bestimmt. Die Präzision dieser Methode erwies sich als gering. Es wurden außerdem die Lagerungsfähigkeit sowie die Abhängigkeit der Enzymaktivität von Temperatur, pH-Wert und einigen Effektoren untersucht. Die Existenz von mindestens 5 pH-Optima der Sialyltransferase ist als Hinweis auf multiple Formen dieses Enzyms im Serum anzusehen.

\section{Analysis of sialyltransferase activity in human serum}

Summary: The activity of sialyltransferase in human serum was determined by a radiochemical assay with desialylized fetuin as acceptor. The precision of this method proved to be low. The effect of storage, temperature, concentration of hydrogen ions and other substances was investigated. As at least $5 \mathrm{pH}$-optima were found, multiple forms of sialyltransferase may well exist in serum.

\section{Einleitung}

Ganzinger et al. (1) empfehlen die Bestimmung der Serumsialyltransferase (EC 2.4.99.1) als neue Kenngröße in der Tumordiagnostik und -verlaufskontrolle. Bisher liegen über Grundlagen der Aktivitätsbestimmung der Sialyltransferase im menschlichen Serum nur sporadische Ergebnisse vor.

Die vorliegende Arbeit schildert unsere Erfahringen über den Einfluß von Lagerung, pH-Wert, Temperatur und einigen Effektoren auf die Aktivitätsbestimmung der Sialyltransferase im menschlịchen Serum, sowie über die Präzision der Bèstimmungsmethode. Die Einschätzung des Wertes dieser Enzymaktivitätsbestimmung für die Malignomdiagnostik wird anhand von Literaturangaben diskutièrt.

\section{Material und Methoden}

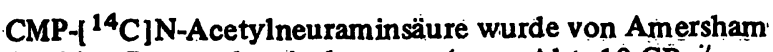
Buchler, Braunschweig, bezogen (spez. Akt. $10 \mathrm{GBq} / \mathrm{mmol}$ ). Fetuin (Sigma München) wurde 1 Stunde in $0,05 \mathrm{~mol} / \mathrm{l}$ $\mathrm{H}_{2} \mathrm{SO}_{4}$-Lösung bei $80^{\circ} \mathrm{C}$ hydrolysiert. Freie Neuraminnsäure und Asialofetuin wurden durch Dowex 1 × 8-Ionenaustauscher
(Serva, Heidelberg) getrennt. Das Asialofetuin wurde gefriergetrocknet und bei Bedarf als $1 \mathrm{mmol} / 1$ Lösung verwandt. Alle übrigen Substanzen stammten von E. Merck, Darmstadt. Die Un̈tersuchung der Sialyltransferase im Serum erfolgte nie später als 4 Stunden nach Blutentnahme. Nach Entnahme und Gerinnung wurde das Blut 10 Minuten bei $3000 \mathrm{~g}$ zentrifugiert. Für die Versuche wurde z. T. Poolserum, z. T. Serum von einzelnen Personen benutzt. Das Serum enthielt keine Zusätze.

Die Messung der Sialyltransferase-Aktivität erfolgte in einem radiochemischen Assay mit desialyliertem Fetuin als Akzeptor. Der Inkubationsansatz enthielt - wenn nicht anders beschrieben - in einem Gesamtvolumen von $210 \mu \mathrm{l}: 0,1 \mu \mathrm{mol}$ Asialofetuin; $5,0 \mu$ mol Trismaleat (pH-Wert 7,3), $0,48 \mathrm{nmol} \mathrm{CMP-}\left[{ }^{14} \mathrm{C}\right] \mathrm{N}-$ Acetylneuraminsäure sowie $100 \mu 1$ Serum. Die Reaktion wurde nach einer Inkubation von 30 Minuten bei $37^{\circ} \mathrm{C}$ mit $2,3 \mu \mathrm{mol} \mathrm{HgCl}{ }_{2}$ gestoppt. Die weitere Aufarbeitung des Ansatzes erfolgte nach der Methode von Bauer et al. (2).

\section{Ergebnisse}

\section{Präzision der Bestimmungsmethode}

Die Präzision der Enzymaktivitätsmessung wurde von uns als Präzision in der Serie und als Präzision von Tag zu Tag bestimmt. Wie Tabelle 1 zeigt, beträgt der Variationskoeffizient in der Serie 13,8\% bzw. 24\%, der Variationskoeffizient von Tag zu Tag $28 \%$. 
Tab. 1. Präzision der Sialyltransferaseaktivitätsmessung.

\begin{tabular}{|c|c|c|c|}
\hline \multicolumn{2}{|c|}{$\begin{array}{l}\text { Mehrfachbestimmung der } \\
\text { Sialyltransferaseaktivität aus } \\
\text { einem Serum, Messung in } \\
\text { einer Serie (Imp/min X ml } \\
\text { Ansatzvolumen) }\end{array}$} & \multicolumn{2}{|c|}{$\begin{array}{l}\text { Sialyltransferaseaktivitäten eines } \\
\text { Serum, an verschiedenen Tagen } \\
\text { gemessen (Imp/min } \times \mathrm{ml} \\
\text { Ansatzvolumen) }\end{array}$} \\
\hline $\begin{array}{r}12420 \\
9720 \\
10940 \\
16400 \\
14830 \\
9390 \\
8680 \\
10460\end{array}$ & $\begin{array}{r}9150 \\
11200 \\
10540 \\
12550\end{array}$ & $\begin{array}{r}12420 \\
10460 \\
7880 \\
6990 \\
12900 \\
8150 \\
9490 \\
4740 \\
6940 \\
7970\end{array}$ & $\begin{array}{r}12320 \\
8950 \\
7800 \\
12070 \\
7640 \\
15030\end{array}$ \\
\hline $\begin{aligned} & =11499 \\
& =2711 \\
V K & =23,6 \%\end{aligned}$ & $\begin{array}{l}\bar{x}=10678 \\
s=1479 \\
V K=13,85 \%\end{array}$ & $\begin{array}{l}\bar{x}=8624 \\
\mathrm{~s}=2382 \\
\mathrm{VK}=27,6 \%\end{array}$ & $\begin{aligned} \bar{x} & =10596 \\
\mathrm{~s} & =3121 \\
\mathrm{VK} & =27,9 \%\end{aligned}$ \\
\hline
\end{tabular}

\section{Linearität der Bestimmungsmethode}

Während eines Beobachtungszeitraumes von $40 \mathrm{~min}$ besteht eine lineare Beziehung zwischen Substratumsatz und Inkubationszeit. Auch die Substratumsatzgeschwindigkeit der Sialyltransferase ist in dem geprüften Bereich von $20 \mu \mathrm{l}$ bis $100 \mu \mathrm{l}$ Serum pro Inkubationsansatz der Enzymkonzentration direkt proportional.

\section{pH-Optimum und Temperaturoptimum der Sialyltrans- feraseaktivität}

Die Abhängigkeit der Sialyltransferaseaktivität im Blutserum von der Wasserstoffionenkonzentration des Testansatzes wurde innerhalb eines $\mathrm{pH}$-Bereichs von $\mathrm{pH} 5,1$ bis $\mathrm{pH} \mathrm{8,6}$ untersucht. Auffälligerweise gibt es kein einheitliches pH-Optimum, sondern mindestens fünf unterschiedliche pH-Optima. Die in Abbildung 1 dargestellte Beziehung zwischen Enzymaktivität und pH-Wert wurde von uns mehrfach reproduziert.

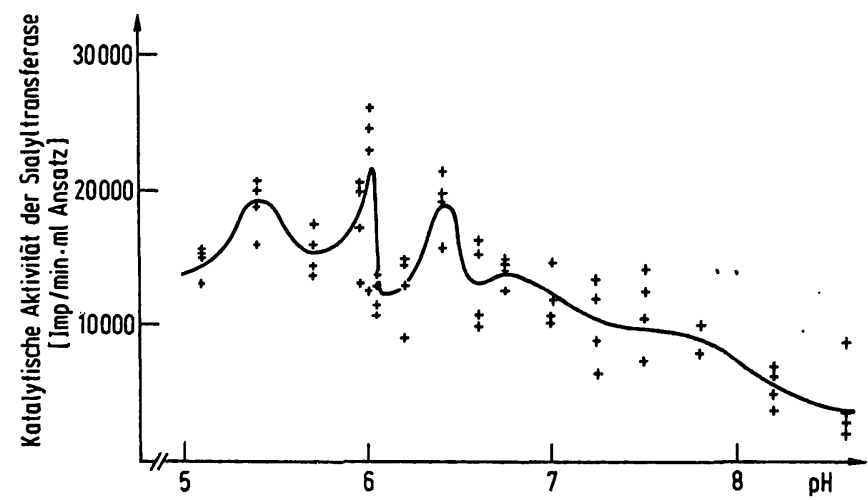

Abb. 1. Abhängigkeit der Sialyltransferaseaktivität im Blutserum von der Wasserstoffionenkonzentration im Testansatz. Die Aktivitätsbestimmung erfolgte im Vierfachansatz.
Bis zu einer Temperatur von $39{ }^{\circ} \mathrm{C}$ nimmt die Substratumsatzgeschwindigkeit mit steigender Inkubations. temperatur $\mathrm{zu}$, bei noch höheren Temperaturen tritt eine Verminderung der Substratumsatzgeschwindigkeit auf.

\section{Einfluß verschiedener Stoffe auf die Sialyltransferase- aktivität}

Magnesium in einer Konzentration von 2 bis $5 \mu \mathrm{mol} /$ Ansatz steigert die Enzymaktivität um 30 bzw. 50\%.

Zusatz von $10 \mu \mathrm{mol}$ Calcium oder $10 \mu \mathrm{mol}$ Mangan zum Ansatz vermindert die Enzymaktivität auf 30 bzw. $60 \%$ des Kontrollwertes.

In Gegenwart von $5 \mu \mathrm{mol} /$ Ansatz Kupfer-, Zink- oder Quecksilberionen wird die Enzymaktivität nahezu vollständig gehemmt. $5 \mu \mathrm{mol} /$ Ansatz Bleiionen haben dagegen keinen Einfluß auf die Enzymaktivität. Da die Sialyltransferaseaktivität von einigen Autoren nur im Blutplasma bestimmt wird, gingen wir der Frage nach, welchen Einfluß gerinnungshemmende Substa':zen auf die Enzymaktivität haben.

Von den von uns untersuchten Antikoagulatien (Heparin, EDTA und Citrat) besitzt besonders das Heparin einen starken Hemmeffekt auf die Sialyltransferaseaktivität. In Anwesenheit von 2, 7,5 und 15 Heparin-Einheiten/ Ansatz wird das Enzym bis auf eine Restaktivität von etwa $20 \%$ gehemmt. Der Zusatz von 0,4 $\mu \mathrm{mol} /$ Ansatz EDTA oder $5 \mu \mathrm{mol} /$ Ansatz Citrat führt zu einer Verminderung der Sialyltransferaseaktivität auf $60 \%$ der Ausgangsaktivität.

ATP in Konzentrationen von 0,05, 0,075 bzw. 0,15 $\mu \mathrm{mol} /$ Ansatz hemmt die Sialyltransferaseaktivität 60 , 70 bzw. 80\%. Bei Zusatz des nicht-ionischen Detergens Triton X 100 (1, 3, $5 \mathrm{~g} / 1$ Ansatz) oder Dithiothreitol $(0,025,0,05,0,1 \mu \mathrm{mol} /$ Ansatz $)$ ändert sich die Sialyltransferaseaktivität nicht.

\section{Stabilität der Sialyltransferaseaktivität im Blutserum}

2-tägige Lagerung des Blutserums bei Raumtemperatur bzw. 84-tägige Lagerung bei Kühlschranktemperatur oder bei $-40^{\circ} \mathrm{C}$ haben keinen Einfluß auf die Sialyltransferaseaktivität. Lagerung des Serums bei $+37^{\circ} \mathrm{C}$ vermindert die Enzymaktivität nach 24 Stunden auf 50\% und nach 48 Stunden auf 20\% der Ausgangsaktivität.

\section{Diskussion}

Die erforderliche direkte Proportionalität zwischen Substratumsatzgeschwindigkeit und Enzymkonzentration ist unter den hier angewandten. Testbedingungen gewährleistet und entspricht den Angaben in der Literatur für ähnliche Testsysteme unter Verwendung verschiedener Substrate und Testbedingungen $(3,4,5,6))^{\prime \prime}$ 
Wegen der möglichen Freisetzung von Sialyltransferase aus Thrombocyten verwenden einige Autoren Plasma als Probe, wobei jedoch die Wahl des Antikoagulans kritisch wird $(6,7,8)$. Wegen des Hemmeffektes aller von uns getesteten Antikoagulantien entschieden wir uns für Serum als Probenmaterial.

Die Untersuchung des Einflusses verschiedener Substanzen auf die Enzymaktivität, die außer der aktivierenden Wirkung von $\mathrm{Mg}^{2+}$ eine deutliche Hemmung durch alle sonstigen getesteten Kationen zeigt, ergab mit diesbezüglichen Angaben in der Literatur für menschliche Sialyltransferase übereinstimmende Resultate (9). Die aus dem Lagerungsversuch resultierende hohe Stabilität der Sialyltransferase (siehe auch 1.c. (10) und 1.c. (11)) bei $22^{\circ} \mathrm{C}, 4^{\circ} \mathrm{C}$ und $-40^{\circ} \mathrm{C}$ erleichtert das Arbeiten mit dem Enzym wesentlich und verringert die Möglichkeit von Lagerungsartefakten. Die graphische Darstellung der Enzymaktivität in Abhängigkeit vom pH-Wert zeigt einen weiten, flachen Verlauf im Bereich von pH 5,1 bis 8,6 mit mehreren Maxima, das höchste bei pH 6,0. Dieser Verlauf, der mehrfach reproduziert werden konnte, ist ein Hinweis auf die Existenz multipler Formen der Sialyltransferase im Serum. Weitere Hinweise auf multiple Formen sind bei Liu et al. $(12,13)$, Alhadeff et al. (9), Kessel et al. (7) und in der Serie von Carlson et al. $(3,4)$ sowie Bartholomew et al. $(5)$ beschrieben.

Als wichtiger nachteiliger Befund erscheint uns die niedrige Präzision der Methode, besonders im Zusammenhang mit den geringen Aktivitätsunterschieden zwischen Tumorkranken und Gesunden. Selbst bei dem großen Aufwand von 8 Parallelansätzen, wie bei Ganzinger et al. (1) beschrieben, ergibt sich bei nur etwa $30 \%$ der Tumorkranken eine Erhöhung der Sialyltransferase im Serum über den Normalbereich von \pm 3 S.D., und diese liegen zum überwiegenden Teil innerhalb des 1,5fachen der oberen Normalbereichsgrenze von 3 S.D. Umfassende Angaben über die Sensitivität und Spezifität der Sialyltransferase im Serum sind uns für maligne Krankheiten noch nicht bekannt. Aus einzelnen Literaturangaben über eine Erhöhung des Enzyms nach partieller Hepatektomie (14), bei verschiedenen Leberkrankheiten (11) im Serum von Polyarthritispatienten (6) und angesichts der weiten Verbreitung von Sialyltransferasen in zahlreichen Geweben (3) läßt sich kaum eine hohe Spezifität der Gesamt-Sialyltransferase im Serum für maligne Prozesse und ihre Anwendung als Tumormarker erhoffen.

Die Sensivität ist aufgrund von Beobachtung erniedrigter Sialyltransferasewerte in Hepatomzellen gegenüber gesunden Kontrollzellen (2) ebenfalls nicht sehr hoch zu erwarten, was auch aus den Daten der Patientenserie von Ganzinger et al. (1) entnommen werden kann.

Wegen der mäßigen Präzision im Zusammenhang mit der niedrig zu veranschlagenden Sensitivität und Spezifität der Bestimmung der Gesamt-Sialyltransferaseaktivität im Serum halten wir zum jetzigen Zeitpunkt eine Einfuihrung des Tests in die Routinediagnostik der malignen Tumoren für nicht angebracht. Zur vollständigen Beurteilung des diagnostischen Wertes der Bestimmung der Sialyltransferase im Serum wird eine vielseitige klinische Studie mit Angabe der Sensitivität und Spezifität des Testes für maligne Krankheiten erforderlich sein.

Eine lohnende Möglichkeit, die Spezifität und Sensitivität des Testes zu verbessern, sehen wir in der weiteren Bearbeitung des Gebietes der multiplen Formen der Sialyltransferasen.

\section{Literatur}

1. Ganzinger, U., Dorner, F., Unger, F. M., Moster, K. \& Jentzsch, K. (1977), Klin. Wochenschr. 55, 553-555.

2. Bauer, C. H., Vischer, P. Grünholz, H. J. \& Reutter, W. (1977) Cancer Res. 37, 1513-1518.

3. Carlson, D. M., Jourdian, G. W. \& Roseman, S. (1973), J. Biol. Chem. 248, 5742-5750.

4. Carlson, D. M., MacGuire, E. J., Jourdian, G. W. \& Roseman, S. (1973), J. Biol. Chem. 248, 5763-5773.

5. Bartholomew, B. A., Jourdian, G. W. \& Roseman, S. (1973), J. Biol. Chem. 248, 5751-5762.

6. Kessel, D. \& Allen, J. (1975), Cancer. Res. 35, 670-672.

7. Kessel, D., Chou, T.-H. \& Allen, J. (1978), Eur. J. Biochem. 82, 535-541.

8. Kim, Y. S., Perdomo, J., Bella, A. \& Nordberg, J. (1971), Biochim. Biophys. Acta 244, 505-512.

9. Alhadeff, J. A., Cimino, G., Janowsky, A. \& O'Brien, J. S. (1977), Biochim. Biophys. Acta 484, 307-321.

10. Van den Eijnden, D. H. \& van Dijk, W. (1974), Biochim. Biophys. Acta 362, 136-149.

11. Mookerjea, S., Michaelis, M. A., Hudgin, R. L., Moscarello, M. A., Chow, A. \& Schachter, H. (1972), Can. J. Biochem. $50,738-740$.

12. Liu, C.-K., Schmied, R. \& Waxman, S. (1979), Clin. Chim. Acta 98, 225-233.

13. Liu, C.-K., Schmied, R., Greenspan, E. M. \& Waxman, S. (1978), Biochim. Biophys. Acta 522, 375-384.

14. Serafini-Cessi, F. (1977), Biochein. J. 166, 381-386.

Dr. Andreas Wilhelm Institut für Arterioskleroseforschung an der Universität Münster Westring 3 D-4400 Münster 
. 